\title{
Qualified and Validated Analytical Procedures for Pharmaceutical Products Majoring from Biological Origins
}

\section{Hideharu Shintani*}

Faculty of Science and Engineering, Chuo University, Tokyo, Japan

In case the technologies were developed that allowed biological or biotechnological materials to be produced in large quantities for pharmaceutical use, the opinion was heard in both sides of industry and regulatory circles that "the process is the product". This perspective was based on the understanding that the main production agents produce large quantities of chemically similar materials that must undergo a variety of separation steps that can select the greatest yield of the highest purity of a desired molecular entity from the cellular-derived milieu. The separation processes used to sort out the one or more target proteins from other, often co-purifying, proteins are optimized, scaled, and validated to reliably achieve the same population of molecular entities from each batch of biologically produced material. The nature of these separation processes is such that even subtle changes in some steps can impart significant variations in the resulting population of proteins. It has been shown repeatedly that successfully defining and controlling the process can define and control the product, hence the rationale for the phrase [1].

Concurrent with advances in process technologies, analytical technologies have also been emerging that allow biological and biotechnological materials to be scrutinized in ever more sensitive and specific physiochemical and functional detail. Increasing attention is being given to the tremendous value to be derived from adequate analytical characterization of protein products and intermediate materials. During early product development, in-depth, orthogonal biochemical information gained on the target molecular entity provides a better understanding of the attributes of the product that may contribute to its efficacy. It also allows an assessment of intrinsic product- or process-related impurities that could impact product safety in early clinical trials. As development proceeds and product batches are manufactured, analytical characterization can be used to obtain biomolecular profiles of the product and its impurities in order to evaluate process consistency. Biomolecular characterization techniques are also used to determine the physiochemical comparability of product batches before and after a process change, to assess the success of process scale-up or scale-down, or following technology transfer of the production to a different manufacturing facility.

In that sense, efforts directed toward the analysis and characterization of the biologica1/biotechnological product has recently achieved a level of significance that was previously considered necessary only for the purification process. A term now widely used in the biotechnology industry is well-characterized biological/biotechnological products or WCBPs (Well Characterized Biotechnology Pharmaceuticals, now formally designated by the FDA as specified products) [2]. The term reflects the important role of rigorous analytics in ensuring safe and effective products, as has been demonstrated in numerous case studies and discussions at industry-regulatory WCBP meetings.

The structural complexity of even simple proteins requires the use of several different analytical technologies to generate a characteristic product "profile". Many of these techniques utilize complex materials and reagents that are relatively labile and subject to manufacturing variability. These features can impact method robustness and the establishment of realistic method performance specifications. There are many factors that should be considered when selecting, optimizing, and validating analytical test methods, and in using test results to establish appropriate specifications. Choices are made during the development cycle regarding the types of standard and state-of-the-art technologies that may be suitable for use with the product. Current regulatory guidance documents and several biotechnology industry publications give considerable information on the analytical methods used with different types of WCBPs and other biological products and current expectations for product characterization, release, and stability testing. Practical considerations should also be factored into the selection of the methods that will be used for routine quality control testing of product batches. Quality control (QC) analytical methods must be robust enough to function reliably over time under varying operational conditions. Failure to fully understand the details of the analytical technology and failure to define the intended application of the method are prime reasons for methods that end up in QC laboratories unable to reliably perform to expectations. The final purpose of the above description is for the sake of obtaining the benefit to human being taking the biological or synthetic pharmaceutical products in safe.

\section{References}

1. (2002) Comparability Studies for Human Plasma-Derived Therapeutics FDA for Biologics Evaluation and Research and Plasma Protein Therapeutic Association Meeting, Rockville.

2. (1996) Elimination of Establishment License Application for Specified Biotechnology and Specified Synthetic Biological Products, Federal Register 61: 24227-24233.
*Corresponding author: Hideharu Shintani, Faculty of Science and Engineering, Chuo University, 1-13-27, Kasuga, Bunkyo, 112-8551, Tokyo, Japan, Tel: +81425922336; Fax +81425922336; E-mail: shintani@mail.hinocatv.ne.jp

Received April 16, 2014; Accepted April 17, 2014; Published April 30, 2014

Citation: Shintani H (2014) Qualified and Validated Analytical Procedures for Pharmaceutical Products Majoring from Biological Origins. Pharmaceut Reg Affairs 3: e131. doi:10.4172/2167-7689.1000e131

Copyright: (c) 2014 Shintani H. This is an open-access article distributed under the terms of the Creative Commons Attribution License, which permits unrestricted use, distribution, and reproduction in any medium, provided the original author and source are credited. 